\title{
A Remark on the Segal-Becker Theorem
}

\author{
Dedicated to Professor Minoru Nakaoka on his 60th birthday
}

By

\author{
Kazumoto KOZIMA*
}

\section{§1. Introduction}

Let $C P^{\infty}$ be the infinite dimensional complex projective space and $B U$ the classifying space of stable complex vector bundles. Then there is the natural inclusion $j: C P^{\infty} \hookrightarrow B U$ and the structure map of the infinite loop space structure defined by the Bott periodicity $\xi: Q(B U) \longrightarrow B U$ where $Q()=\operatorname{Colim}_{n} Q^{n} \Sigma^{n}()$. Let $\lambda: Q\left(C P^{\infty}\right) \longrightarrow$ $B U$ be the composition $\xi \circ Q(j)$. The results of Segal [8] and Becker [2] show us that there exists a map $s: B U \longrightarrow Q\left(C P^{\infty}\right)$ such that $\lambda \circ s=\mathrm{id}$.

The main result of this paper is to show that one can take $s$ satisfying that $s \circ j \simeq$ inclusion : $C P^{\infty} \longrightarrow Q\left(C P^{\infty}\right)$.

To show this, we will use the results of Brumfiel-Madsen [4] for the evaluation of the transfer map.

\section{§2. The Construction of the Splitting}

Let $U(n)$ be the unitary group and $T^{n}$ its maximal torus. Let $N T^{n}$ be the normaliser of $T^{n}$ in $U(n)$. We also define homogeneous spaces of $U(2 n)$ :

$$
E_{n}=U(2 n) / U(n) \text { and } E_{n}^{\prime}=U(n+1) / U(n) \text {. }
$$

Then the construction of splitting in [2] can be reformulated as follows.

Let $r: Q\left(X_{+}\right) \longrightarrow Q(X)$ be the map induced by the canonical projection and $a: Q(X) \longrightarrow Q\left(X_{+}\right)$the right adjoint of $r$. Let $t_{n}: E_{n} / U(n)_{+}$

Communicated by N. Shimada, May 10, 1983.

* Department of Mathematics, Kyoto University, Kyoto 606, Japan. 
$\longrightarrow Q\left(E_{n} / N T_{+}^{n}\right)$ the Becker-Gottlieb transfer ([2], [3]) associated with the smooth fiber bundle

$$
U(n) / N T^{n} \longrightarrow E_{n} / N T^{n} \longrightarrow E_{n} / U(n) .
$$

$E_{n} / T^{n}$ has the action of $N T^{n} / T^{n}=\Sigma_{n}$ which sends $e T^{n}$ to $e n T^{n}$ where $e \in E_{n}$ and $n \in N T^{n} . \quad(X)^{n}$ is also a $\sum_{n}$-space by the permutation of the coordinates.

Since the elements of $E_{n}$ can be considered as the $n$-frames in $\boldsymbol{C}^{2 n}$, we define a $\Sigma_{n}$-equivariant map

$$
h_{n}: E_{n} / T^{n} \longrightarrow\left(C P^{2 n-1}\right)^{n}
$$

by corresponding each vector to its representative element in $C P^{2 n-1}$.

Also, since $E_{n} / T^{n} \longrightarrow E_{n} / N T^{n}$ is a principal $\Sigma_{n}$-bundle, there is a $\Sigma_{n}$-equivariant map

$$
c_{n}: E_{n} / T^{n} \longrightarrow E \Sigma_{n}
$$

which covers the classifying map of this principal bundle where $E \Sigma_{n}$ is the contractible free $\Sigma_{n}$-space. Thus we obtain a map

$$
k_{n}=\left(c_{n} \times h_{n}\right) / \Sigma_{n}: E_{n} / N T^{n} \longrightarrow\left(E \Sigma_{n} \times\left(C P^{2 n-1}\right)^{n}\right) / \Sigma_{n} .
$$

There is also the Barratt-Quillen map

$$
w_{n}:\left(E_{n} \times(X)^{n}\right) / \Sigma_{n} \longrightarrow Q\left(X_{+}\right) .
$$

Notice that the composition $X \stackrel{i_{1}}{\longrightarrow}\left(E \Sigma_{n} \times(X)^{n}\right) / \Sigma_{n} \stackrel{w_{n}}{\longrightarrow} Q\left(X_{+}\right)$is homotopic to the composition $X \stackrel{\text { incl. }}{\longrightarrow} Q(X) \stackrel{a}{\longrightarrow} Q\left(X_{+}\right)$where $i_{1}$ is the map defined by the equation

$$
i_{1}(x)=\left(*_{E_{n}},\left(x, *_{X}, *_{X}, \cdots, *_{X}\right)\right) \text { for } x \in X .
$$

So the following Lemma is clear.

Lemma 2.1. The composition

$$
C P^{n-1}=E_{n}^{\prime} / S^{1} \longrightarrow E_{n} / N T^{n} \stackrel{w_{n} \circ k_{n}}{\longrightarrow} Q\left(C P^{2 n-1}\right)
$$

is homotopic to the composition $C P^{n-1} \longrightarrow C P_{+}^{2 n-1} \stackrel{\text { inc1 }}{\longrightarrow} Q\left(C P_{+}^{2 n-1}\right)$.

Remark. One can easily show that the composition $w_{n} \circ k_{n}: E_{n} / N T^{n}$ $\longrightarrow Q\left(C P_{+}^{2 n-1}\right)$ agrees with the composition of the Kahn-Priddy pretransfer $t: E_{n} / N T^{n} \longrightarrow Q\left(E_{n} / N T^{n-1} \times S_{+}^{1}\right)$ associated with the $n$-fold covering $E_{n} / N T^{n-1} \times S^{1} \longrightarrow E_{n} / N T^{n}$ and the map $Q\left(E_{n} / N T^{n-1} \times S_{+}^{1}\right)$ $\longrightarrow Q\left(C P_{+}^{2 n-1}\right)$ which is induced from the quotient map. (Compare 
$[6],[7]$.

Now we are ready to define the splitting $s$. Let us consider the composition

$$
\begin{aligned}
& s_{n}: E_{n} / U(n) \stackrel{\longrightarrow}{\longrightarrow} E_{n} / U(n)_{+} \stackrel{t_{n}}{\longrightarrow} Q\left(E_{n} / N T_{+}^{n}\right) \stackrel{Q\left(w_{n}{ }^{\circ} k_{n+}\right)}{\longrightarrow} Q Q\left(C P_{+}^{2 n-1}\right) \\
& \stackrel{\zeta}{\longrightarrow} Q\left(C P_{+}^{2 n-1}\right) \stackrel{r}{\longrightarrow} Q\left(C P^{2 n-1}\right)
\end{aligned}
$$

where $w_{n} \circ k_{n+}$ is the pointed extension of $w_{n} \circ k_{n}$ and $\zeta$ is the structure map of the infinite loop space $Q\left(C P_{+}^{2 n-1}\right)$. As in [2] and [9], $t_{n}$ is compatible with $n$. So, since all the constructions are compatible with $n$, by taking the limit, we obtain $s: B U \longrightarrow Q\left(C P^{\infty}\right)$.

\section{§3. The Proof of the Main Result}

By virtue of (2.1), we have only to prove that the diagram

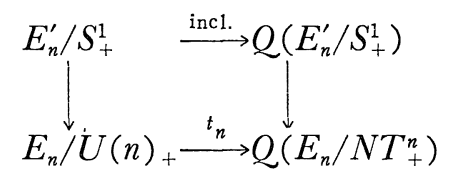

commutes up to homotopy where the virtical maps are induced from the inclusion $E_{n}^{\prime c} \longleftrightarrow E_{n}$.

We need the evaluation of the transfer.

Proposition 3.1. The following diagram is homotopy commutative;

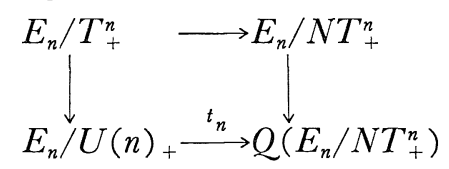

where the maps with no name are induced from the canonical projections.

This proposition is a corollary of Brumfiel and Madsen [4]. (See Theorem 3.5 of [4].)

Since the diagram

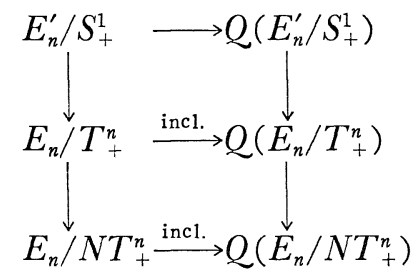


commutes up to homotopy, we get the main result:

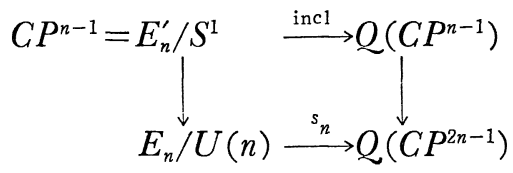

commutes up to homotopy.

Thus $s \circ j$ is homotopic to the canonical inclusion as an element of $\lim _{n} \operatorname{Map}\left(C P^{n}, Q\left(C P^{\infty}\right)\right)$. Then $\lambda \circ s \circ j$ is homotopic to $j$ on the finite skeleton. So one can easily show that $\lambda \circ s: B U \longrightarrow B U$ induces identities on the $K$-homology groups and on the $K$-cohomology groups, by using the fact that $s$ is an $H$-map. (See [9].) Thus our $s$ is a splitting.

Let $P^{m}()$ be the $m$-th term of the cohomology defined by $Q\left(C P^{\infty}\right)$. Then we have the Milnor exact sequence

$$
0 \longrightarrow \lim _{n}^{1} P^{-1}\left(C P^{n}\right) \longrightarrow P^{0}\left(C P^{\infty}\right) \longrightarrow \lim _{n} P^{0}\left(C P^{n}\right) \longrightarrow 0 \text {. }
$$

As in [5], one can easily prove that $P^{-1}\left(C P^{n}\right)$ is finite. So $\lim ^{1}$ term vanishes and we have the main theorem:

\section{Theorem 3.2. The composition}

$$
s \circ j: C P^{\infty} \longrightarrow B U \longrightarrow Q\left(C P^{\infty}\right)
$$

is homotopic to the canonical inclusion.

\section{References}

[1] Adams, J. F., Infinite loop space, Annals of mathematics studies, 90, Princeton Univ. Press, 1978.

[2] Becker, J. C., Characteristic classes and $K$-theory, Lecture Notes in Math., 428, Springer, 1973, 132-143.

[3] Becker, J. C. and Gottlieb, D. H., The transfer map and fibre bundles, Topology, 14 (1975), 1-12.

[4] Brumfiel, G. W. and Madsen, I., Evaluation of the transfer and the universal surgery classes, Inventiones math., 32 (1976), 133-169.

[5] Kono, A., A note on the Segal-Becker type splittings, to appear in J. Math. Kyoto Univ.

[6] Kahn, D. S. and Priddy, S. B., Applications of the transfer to stable homotopy theory, Bull. A. M. S., 78(6) (1976), 981-987.

[7] Roush, F. W., Transfer in generalized cohomology theories, Thesis, Princeton, 1971.

[8] Segal, G. B., The stable homotopy of complex projective space, Quart. J. Math. Oxford (2), 24 (1973), 1-5.

[9] Snaith, V. P., Algebraic cobordism and K-theory, Memoirs of the A. M. S., vol. 21, no. 211, 1979. 